\title{
Limit theorems for locally stationary processes
}

\section{Rafael Kawka ${ }^{1}$}

Received: 10 September 2019 / Revised: 10 June 2020 / Published online: 1 October 2020 (c) The Author(s) 2020

\section{Abstract}

We present limit theorems for locally stationary processes that have a one sided timevarying moving average representation. In particular, we prove a central limit theorem (CLT), a weak and a strong law of large numbers (WLLN, SLLN) and a law of the iterated logarithm (LIL) under mild assumptions using a time-varying BeveridgeNelson decomposition.

Keywords Locally stationary process - Central limit theorem · Law of large numbers $\cdot$ Law of the iterated logarithm

\section{Introduction}

In this paper we consider locally stationary processes, defined via a triangular sequence of stochastic processes $\left\{\eta_{t, T}\right\}_{t=1, \ldots, T}$ with $T \in \mathbb{N}$, where every $\eta_{t, T}$ has a representation of the form

$$
\eta_{t, T}=\mu\left(\frac{t}{T}\right)+\sum_{j=0}^{\infty} \psi_{j, t, T} \varepsilon_{t-j}, \quad t=1, \ldots, T .
$$

Throughout this paper we impose the following assumption on the error sequence $\left\{\varepsilon_{t}\right\}_{t \in \mathbb{Z}}$, on the moving average coefficients $\psi_{j, t, T}$ and on the trend function $\mu$.

Assumption 1.1 The random variables $\left\{\varepsilon_{t}\right\}_{t \in \mathbb{Z}}$ are independent and identically distributed with $\mathbb{E} \varepsilon_{t}=0, \mathbb{E} \varepsilon_{t}^{2}=1$ and $\mathbb{E}\left|\varepsilon_{t}\right|^{2+\kappa}<\infty$ for some $\kappa>0$. The coefficients $\psi_{j, t, T}$ in the moving average representation (1) fulfill

$$
\sup _{t, T}\left|\psi_{j, t, T}\right| \leq \frac{K}{l(j)},
$$

Rafael Kawka

kawka@statistik.tu-dortmund.de

1 Fakultät Statistik, Technische Universität Dortmund, Vogelpothsweg 87, 44227 Dortmund, Germany 
with constant $K$ independent of $T$ and some positive deterministic sequence $\{l(j)\}_{j \in \mathbb{N}_{0}}$ satisfying

$$
\sum_{j=0}^{\infty} \frac{j}{l(j)}<\infty
$$

The trend function $\mu:[0,1] \rightarrow \mathbb{R}$ is assumed to be bounded and continuous almost everywhere.

Remark 1.2 In contrast to the definition of Dahlhaus and Polonik (2006) we restrict locally stationary processes to have a one-sided moving average representation. Nonetheless, our definition covers most of the important examples of locally stationary processes. For instance, it follows from Dahlhaus and Polonik (2009, Proposition 2.4) that time-varying causal ARMA processes have a representation of the form (1).

The idea behind locally stationary processes is that, after rescaling the time domain to the unit interval, the process can be approximated locally in time by a stationary process. Therefore, one usually assumes that $\psi_{j, t, T} \approx \psi_{j}(t / T)$ for some well behaving functions $\psi_{j}$.

Assumption 1.3 There exist functions $\psi_{j}:[0,1] \rightarrow \mathbb{R}$ with

$$
\begin{aligned}
\left\|\psi_{j}\right\|_{\infty} & \leq \frac{K}{l(j)}, \\
V\left(\psi_{j}\right) & \leq \frac{K}{l(j)}
\end{aligned}
$$

and

$$
\sum_{t=1}^{T}\left|\psi_{j, t, T}-\psi_{j}\left(\frac{t}{T}\right)\right| \leq \frac{K}{l(j)}, \quad \text { for all } T \in \mathbb{N}
$$

where $V(f)$ denotes the total variation of a function $f$ on $[0,1]$.

Remark 1.4 The coefficient functions are uniquely defined almost everywhere. To see this let $\left\{\eta_{t, T}\right\}_{t=1, \ldots, T}$ be locally stationary process with moving average coefficients $\psi_{j, t, T}$ and corresponding coefficient functions $\psi_{j}$. Let $\phi_{j}$ be another set of coefficient functions that fulfills Assumption 1.3. Then it holds that

$$
\begin{aligned}
\left\|\psi_{j}-\phi_{j}\right\|_{L^{1}} & =\lim _{T \rightarrow \infty} \frac{1}{T} \sum_{t=1}^{T}\left|\psi_{j}\left(\frac{t}{T}\right)-\phi_{j}\left(\frac{t}{T}\right)\right| \\
& \leq \lim _{T \rightarrow \infty} \frac{1}{T}\left\{\sum_{t=1}^{T}\left|\psi_{j, t, T}-\psi_{j}\left(\frac{t}{T}\right)\right|+\sum_{t=1}^{T}\left|\psi_{j, t, T}-\phi_{j}\left(\frac{t}{T}\right)\right|\right\} \\
& \leq \lim _{T \rightarrow \infty} \frac{2 K}{T l(j)}=0,
\end{aligned}
$$

implying $\psi_{j}=\phi_{j}$ almost everywhere. 
For every $u \in[0,1]$ we define the process $\left\{\eta_{t}(u)\right\}_{t \in \mathbb{Z}}$ via

$$
\eta_{t}(u)=\mu(u)+\sum_{j=0}^{\infty} \psi_{j}(u) \varepsilon_{t-j} .
$$

By Assumption 1.3 the centered process $\left\{\eta_{t}(u)-\mu(u)\right\}_{t \in \mathbb{Z}}$ is weakly stationary with long-run variance given by $\Psi^{2}(u)$, where

$$
\Psi(u)=\sum_{j=0}^{\infty} \psi_{j}(u) .
$$

The main purpose of the process $\left\{\eta_{t}(u)\right\}_{t \in \mathbb{Z}}$ is to approximate $\left\{\eta_{t, T}\right\}_{t=1, \ldots, T}$. In particular, the process $\left\{\eta_{t, T}\right\}_{t=1, \ldots, T}$ should approximately behave like the stationary process $\left\{\eta_{t}(u)\right\}_{t \in \mathbb{Z}}$ in the rescaled time point $u=t / T$. For brevity, we define the auxiliary process $\left\{\tilde{\eta}_{t, T}\right\}_{t=1, \ldots, T}$ via $\tilde{\eta}_{t, T}=\eta_{t}(t / T)$, i.e.

$$
\tilde{\eta}_{t, T}=\mu\left(\frac{t}{T}\right)+\sum_{j=0}^{\infty} \psi_{j}\left(\frac{t}{T}\right) \varepsilon_{t-j} .
$$

Under the stated assumptions it holds that (cf. Lemma A.1 in the appendix)

$$
\frac{1}{T} \sum_{t=1}^{T}\left(\eta_{t, T}-\tilde{\eta}_{t, T}\right) \stackrel{P}{\rightarrow} 0
$$

as $T \rightarrow \infty$. Hence, the process $\left\{\eta_{t}(u)\right\}_{t \in \mathbb{Z}}$ approximates the locally stationary process on average over all rescaled time points $1 / T, 2 / T \ldots, 1$. Later we will strengthen condition (2) in order to obtain a stronger approximation.

Remark 1.5 The construction of locally stationary processes with time dependent moving-average coefficients $\psi_{j, t, T}$ on the one hand and approximating functions $\psi_{j}$ on the other hand looks cumbersome at first glance. It seems more natural to define locally stationary processes directly via (3). However, it was already pointed out by Künsch (1995) and Dahlhaus and Polonik (2009) that this rules out interesting examples such as time-varying autoregressive processes.

Consider the stationary approximating process $\left\{\eta_{t}(u)\right\}_{t \in \mathbb{Z}}$ for some fixed $u \in[0,1]$. By the Beveridge-Nelson decomposition (cf. Phillips and Solo 1992) it holds that

$$
\begin{aligned}
\eta_{t}(u) & =\mu(u)+\sum_{j=0}^{\infty} \psi_{j}(u) \varepsilon_{t-j} \\
& =\mu(u)+\sum_{j=0}^{\infty} \psi_{j}(u) \varepsilon_{t}-\sum_{j=0}^{\infty}\left(\sum_{k=j+1}^{\infty} \psi_{k}(u)\right)\left(\varepsilon_{t-j}-\varepsilon_{t-1-j}\right),
\end{aligned}
$$


which is well defined due to Assumption 1.3. Setting $u=t / T$ we obtain a time-varying Beveridge-Nelson decomposition for the auxiliary process $\left\{\tilde{\eta}_{t, T}\right\}_{t=1, \ldots, T}$.

Lemma 1.6 (Time-varying Beveridge-Nelson decomposition) The auxiliary process $\left\{\tilde{\eta}_{t, T}\right\}_{t=1, \ldots, T}$ exhibits a representation of the form

$$
\tilde{\eta}_{t, T}=\mu\left(\frac{t}{T}\right)+\Psi\left(\frac{t}{T}\right) \varepsilon_{t}-\sum_{j=0}^{\infty} \tilde{\psi}_{j}\left(\frac{t}{T}\right)\left(\varepsilon_{t-j}-\varepsilon_{t-1-j}\right)
$$

with

$$
\tilde{\psi}_{j}(u)=\sum_{k=j+1}^{\infty} \psi_{j}(u)
$$

The time-varying Beveridge-Nelson decomposition will be useful for the derivation of the main results in this paper. In particular, we will use it to generalize the proof techniques of Phillips and Solo (1992) to the locally stationary framework.

\section{Main results}

The first limit theorem we present is a CLT for locally stationary processes. To motivate the outcome, we first derive the result for an easy example. Let $\left\{\eta_{t, T}\right\}_{t=1, \ldots, T}$ be defined by

$$
\eta_{t, T}=\phi\left(\frac{t}{T}\right) \varepsilon_{t}, \quad t=1, \ldots, T,
$$

for some bounded variation function $\phi:[0,1] \rightarrow \mathbb{R}$ and $\left\{\varepsilon_{t}\right\}_{t \in \mathbb{Z}}$ being a sequence of independent and identically $\mathcal{N}(0,1)$ distributed random variables. Then, it holds that

$$
\frac{1}{\sqrt{T}} \sum_{t=1}^{T} \eta_{t, T} \sim \mathcal{N}\left(0, \frac{1}{T} \sum_{t=1}^{T} \phi^{2}\left(\frac{t}{T}\right)\right) .
$$

Since $\phi$ is of bounded variation it is square-integrable on the unit interval and it holds that

$$
\lim _{T \rightarrow \infty} \frac{1}{T} \sum_{t=1}^{T} \phi^{2}\left(\frac{t}{T}\right)=\int_{0}^{1} \phi^{2}(u) d u
$$

and Lévy's continuity theorem implies that

$$
\frac{1}{\sqrt{T}} \sum_{t=1}^{T} \eta_{t, T} \stackrel{d}{\rightarrow} \mathcal{N}\left(0, \int_{0}^{1} \phi^{2}(u) d u\right) .
$$


Note that the approximating stationary process $\left\{\eta_{t}(u)\right\}_{t \in \mathbb{Z}}$ is defined by $\eta_{t}(u)=$ $\phi(u) \varepsilon_{t}$ with long-run variance given by $\phi^{2}(u)$. Hence, the variance of the limiting distribution in (4) is equal to the integrated long-run variance of the auxiliary process. This result also holds for arbitrary centered locally stationary processes.

Theorem 2.1 (CLT) Let $\left\{\eta_{t, T}\right\}_{t=1, \ldots, T}$ be a locally stationary process with movingaverage representation (1) that satisfies Assumptions 1.1 and 1.3. Then, as $T \rightarrow \infty$, it holds that

$$
\frac{1}{\sqrt{T}} \sum_{t=1}^{T}\left\{\eta_{t, T}-\mu\left(\frac{t}{T}\right)\right\} \stackrel{d}{\rightarrow} \mathcal{N}\left(0,\|\Psi\|_{L^{2}}^{2}\right)
$$

where $\|\Psi\|_{L^{2}}$ denotes the $L^{2}$ norm of $\Psi$ on the unit interval.

Proof It suffices to show the claim for the auxiliary process $\left\{\tilde{\eta}_{t, T}\right\}_{t=1, \ldots, T}$ since

$$
\frac{1}{\sqrt{T}} \sum_{t=1}^{T} \eta_{t, T}=\frac{1}{\sqrt{T}} \sum_{t=1}^{T} \tilde{\eta}_{t, T}+\frac{1}{\sqrt{T}} \sum_{t=1}^{T}\left(\eta_{t, T}-\tilde{\eta}_{t, T}\right)
$$

and the second term goes to zero in probability by Lemma A.1. Without loss of generality we assume that $\mu(u)=0$ for all $u \in[0,1]$. By Lemma 1.6 it holds that

$$
\frac{1}{\sqrt{T}} \sum_{t=1}^{T} \tilde{\eta}_{t, T}=\frac{1}{\sqrt{T}} \sum_{t=1}^{T} \Psi\left(\frac{t}{T}\right) \varepsilon_{t}-\frac{1}{\sqrt{T}} \sum_{t=1}^{T} \sum_{j=0}^{\infty} \tilde{\psi}_{j}\left(\frac{t}{T}\right)\left(\varepsilon_{t-j}-\varepsilon_{t-1-j}\right)
$$

We show that the first term in (5) converges in distribution and the second term vanishes in probability. By the i.i.d. assumption on the innovation terms it holds that

$$
\operatorname{Var}\left(\frac{1}{\sqrt{T}} \sum_{t=1}^{T} \Psi\left(\frac{t}{T}\right) \varepsilon_{t}\right)=\frac{1}{T} \sum_{t=1}^{T} \Psi^{2}\left(\frac{t}{T}\right) \rightarrow \int_{0}^{1} \Psi^{2}(u) d u
$$

Next, we verify the Lyapunov condition. By Assumption 1.1 there exists some $\kappa>0$ such that $\mathbb{E}\left|\varepsilon_{t}\right|^{2+\kappa}$ is finite. Hence,

$$
\begin{aligned}
& \lim _{T \rightarrow \infty} \frac{\sum_{t=1}^{T} \mathbb{E}\left|\frac{1}{\sqrt{T}} \Psi\left(\frac{t}{T}\right) \varepsilon_{t}\right|^{2+\kappa}}{\left(\operatorname{Var}\left(\frac{1}{\sqrt{T}} \sum_{t=1}^{T} \Psi\left(\frac{t}{T}\right) \varepsilon_{t}\right)\right)^{1+\kappa / 2}} \\
& =\lim _{T \rightarrow \infty} \frac{\mathbb{E}\left|\varepsilon_{1}\right|^{2+\kappa}}{T^{\kappa / 2}} \lim _{T \rightarrow \infty} \frac{\frac{1}{T} \sum_{t=1}^{T} \Psi^{2+\kappa}\left(\frac{t}{T}\right)}{\left(\frac{1}{T} \sum_{t=1}^{T} \Psi^{2}\left(\frac{t}{T}\right)\right)^{1+\kappa / 2}}=0 .
\end{aligned}
$$


From the Lindeberg-CLT for triangular arrays we deduce that

$$
\frac{1}{\sqrt{T}} \sum_{t=1}^{T} \Psi\left(\frac{t}{T}\right) \varepsilon_{t} \stackrel{d}{\rightarrow} \mathcal{N}\left(0, \int_{0}^{1} \Psi^{2}(u) d u\right)
$$

To finish the proof it remains to show that the second term in (5) goes to zero in probability. It holds that

$$
\begin{aligned}
& \sum_{j=0}^{\infty} \tilde{\psi}_{j}\left(\frac{t}{T}\right)\left(\varepsilon_{t-j}-\varepsilon_{t-1-j}\right)=\sum_{j=0}^{\infty}\left\{\tilde{\psi}_{j}\left(\frac{t}{T}\right) \varepsilon_{t-j}-\tilde{\psi}_{j}\left(\frac{t}{T}\right) \varepsilon_{t-1-j}\right\} \\
& =\sum_{j=0}^{\infty}\left\{\tilde{\psi}_{j}\left(\frac{t}{T}\right) \varepsilon_{t-j}-\tilde{\psi}_{j}\left(\frac{t-1}{T}\right) \varepsilon_{t-1-j}\right. \\
& \left.\quad+\tilde{\psi}_{j}\left(\frac{t-1}{T}\right) \varepsilon_{t-1-j}-\tilde{\psi}_{j}\left(\frac{t}{T}\right) \varepsilon_{t-1-j}\right\} \\
& =\sum_{j=0}^{\infty}\left\{\tilde{\psi}_{j}\left(\frac{t}{T}\right) \varepsilon_{t-j}-\tilde{\psi}_{j}\left(\frac{t-1}{T}\right) \varepsilon_{t-1-j}\right\} \\
& \quad+\sum_{j=0}^{\infty}\left\{\tilde{\psi}_{j}\left(\frac{t-1}{T}\right) \varepsilon_{t-1-j}-\tilde{\psi}_{j}\left(\frac{t}{T}\right) \varepsilon_{t-1-j}\right\} .
\end{aligned}
$$

Taking partial sum of the first term and dividing by $T^{1 / 2}$ leads to:

$$
\begin{aligned}
& \frac{1}{\sqrt{T}} \sum_{t=1}^{T} \sum_{j=0}^{\infty}\left\{\tilde{\psi}_{j}\left(\frac{t}{T}\right) \varepsilon_{t-j}-\tilde{\psi}_{j}\left(\frac{t-1}{T}\right) \varepsilon_{t-1-j}\right\} \\
& =\frac{1}{\sqrt{T}} \sum_{j=0}^{\infty}\left\{\tilde{\psi}_{j}(1) \varepsilon_{T-j}-\tilde{\psi}_{j}(0) \varepsilon_{-j}\right\}
\end{aligned}
$$

as the sum over $t$ is telescopic. Since

$$
\begin{aligned}
\mathbb{E}\left|\sum_{j=0}^{\infty} \sup _{u \in(0,1)}\right| \tilde{\psi}_{j}(u)\left|\varepsilon_{t-j}\right| & \leq \sum_{j=0}^{\infty} \sum_{k=j+1}^{\infty} \sup _{u \in(0,1)}\left|\psi_{k}(u)\right| \mathbb{E}\left|\varepsilon_{1}\right| \\
& \leq \sum_{j=0}^{\infty} \frac{j K \mathbb{E}\left|\varepsilon_{1}\right|}{l(j)}<\infty
\end{aligned}
$$

for an arbitrary $t \in\{1, \ldots, T\}$ it follows that the term on the right hand side of (7) converges to zero in probability. 
It remains to prove that the scaled partial sum of the second term in (6) also vanishes asymptotically. It holds that

$$
\begin{aligned}
& \mathbb{E}\left|\frac{1}{\sqrt{T}} \sum_{t=1}^{T} \sum_{j=0}^{\infty}\left\{\tilde{\psi}_{j}\left(\frac{t}{T}\right)-\tilde{\psi}_{j}\left(\frac{t-1}{T}\right)\right\} \varepsilon_{t-1-j}\right| \\
& \leq \frac{1}{\sqrt{T}} \sum_{t=1}^{T} \sum_{j=0}^{\infty}\left|\tilde{\psi}_{j}\left(\frac{t}{T}\right)-\tilde{\psi}_{j}\left(\frac{t-1}{T}\right)\right| \mathbb{E}\left|\varepsilon_{1}\right| \\
& \leq \frac{1}{\sqrt{T}} \sum_{j=0}^{\infty} V\left(\tilde{\psi}_{j}\right) \mathbb{E}\left|\varepsilon_{1}\right|,
\end{aligned}
$$

which converges to zero if the $V\left(\tilde{\psi}_{j}\right)$ are summable. Using the definition of the total variation we obtain

$$
\begin{aligned}
\sum_{j=0}^{\infty} V\left(\tilde{\psi}_{j}\right) & =\sum_{j=0}^{\infty} \sup _{\substack{0 \leq x_{1}<\ldots<x_{M} \leq 1 \\
M \in \mathbb{N}}} \sum_{i=1}^{M}\left|\tilde{\psi}_{j}\left(x_{i+1}\right)-\tilde{\psi}_{j}\left(x_{i}\right)\right| \\
& \leq \sum_{j=0}^{\infty} \sum_{\substack{k=j+1 \\
0 \leq x_{1}<\ldots<x_{M} \leq 1}}^{\infty} \sum_{i=1}^{M}\left|\psi_{j}\left(x_{i+1}\right)-\psi_{j}\left(x_{i}\right)\right| \\
& =\sum_{j=0}^{\infty} \sum_{k=j+1}^{\infty} V\left(\psi_{j}\right) \\
& \leq \sum_{j=0}^{\infty} \frac{j K}{l(j)},
\end{aligned}
$$

which is finite by Assumption 1.1.

From Theorem 2.1 we immediately obtain a WLLN.

Corollary 2.2 (WLLN) Let $\left\{\eta_{t, T}\right\}_{t=1, \ldots, T}$ be a locally stationary process defined via its moving-average representation (1) with Assumptions 1.1 and 1.3 in place. Then, as $T \rightarrow \infty$, it holds that

$$
\frac{1}{T} \sum_{t=1}^{T}\left\{\eta_{t, T}-\mu\left(\frac{t}{T}\right)\right\} \stackrel{P}{\rightarrow} 0
$$

In order to prove a SLLN and a LIL we require a stronger assumption that connects the coefficient functions $\psi_{j, t, T}$ and the approximating functions $\psi_{j}$. The following assumption, that immediately implies condition (2), corresponds to assumption (69) in Dahlhaus (2012). 
Assumption 2.3 The functions $\psi_{j}$ and the moving average coefficients $\psi_{j, t, T}$ satisfy

$$
\sup _{1 \leq t \leq T}\left|\psi_{j, t, T}-\psi_{j}\left(\frac{t}{T}\right)\right| \leq \frac{K}{T l(j)}, \quad \text { for all } T \in \mathbb{N} \text {. }
$$

Previously, we observed that the stationary process $\left\{\eta_{t}(u)\right\}_{t \in \mathbb{Z}}$ approximates the locally stationary process on average over the series. Under Assumption 2.3 we have a better approximation as it now holds that $\eta_{t, T}-\tilde{\eta}_{t, T}=\mathcal{O}_{P}\left(T^{-1}\right)$. This follows from the fact that

$$
\begin{aligned}
\lim _{T \rightarrow \infty} \mathbb{E}\left|\eta_{t, T}-\tilde{\eta}_{t, T}\right| & \leq \lim _{T \rightarrow \infty} \sum_{j=0}^{\infty}\left|\psi_{j, t, T}-\psi_{j}\left(\frac{t}{T}\right)\right| \mathbb{E}\left|\varepsilon_{t-j}\right| \\
& \leq \lim _{T \rightarrow \infty} \sum_{j=0}^{\infty} \frac{K \mathbb{E}\left|\varepsilon_{0}\right|}{T l(j)}=0 .
\end{aligned}
$$

Consequently, the stationary process $\left\{\eta_{t}(u)\right\}_{t \in \mathbb{Z}}$ approximates the locally stationary process $\left\{\eta_{t, T}\right\}_{t=1, \ldots, T}$ in every rescaled time point $u=t / T$. In fact, we even have the following strong approximation. It holds that

$$
\sup _{1 \leq t \leq T}\left|\eta_{t, T}-\tilde{\eta}_{t, T}\right| \stackrel{\text { a.s. }}{\rightarrow} 0
$$

as $T \rightarrow \infty$ (cf. Lemma A.2 in the appendix).

Theorem 2.4 (SLLN) Let $\left\{\eta_{t, T}\right\}_{t=1, \ldots, T}$ be a locally stationary process defined via its moving-average representation (1) with Assumptions 1.1, 1.3 and 2.3 in place. Then, as $T \rightarrow \infty$, it holds that

$$
\frac{1}{T} \sum_{t=1}^{T} \eta_{t, T} \stackrel{a . s .}{\rightarrow} \int_{0}^{1} \mu(u) d u .
$$

Proof It suffices to show the claim for the auxiliary process $\left\{\tilde{\eta}_{t, T}\right\}_{t=1, \ldots, T}$ since the strong approximation (8) implies that

$$
\frac{1}{T} \sum_{t=1}^{T} \eta_{t, T}=\frac{1}{T} \sum_{t=1}^{T} \tilde{\eta}_{t, T}+o_{a . s .}(1)
$$

The trend function $\mu$ is assumed to be bounded and continuous everywhere. This implies that it is Riemann integrable and we immediately deduce that

$$
\lim _{T \rightarrow \infty} \frac{1}{T} \sum_{t=1}^{T} \mu\left(\frac{t}{T}\right)=\int_{0}^{1} \mu(u) d u
$$


It remains to show that

$$
\frac{1}{T} \sum_{t=1}^{T} \sum_{j=0}^{\infty} \psi_{j}\left(\frac{t}{T}\right) \varepsilon_{t-j} \stackrel{\text { a.s. }}{\rightarrow} 0
$$

Using Lemma 1.6 we first need to verify that

$$
\frac{1}{T} \sum_{t=1}^{T} \Psi\left(\frac{t}{T}\right) \varepsilon_{t} \stackrel{\text { a.s. }}{\rightarrow} 0
$$

It holds that

$$
\sup _{u \in(0,1)}|\Psi(u)| \leq \sup _{u \in(0,1)} \sum_{j=0}^{\infty}\left|\psi_{j}(u)\right| \leq \sum_{j=0}^{\infty} \sup _{u \in(0,1)}\left|\psi_{j}(u)\right| \leq \sum_{j=0}^{\infty} \frac{K}{l(j)}<\infty .
$$

Since the $\varepsilon_{t}$ 's are independent and identically distributed with $\mathbb{E}\left(\varepsilon_{1}\right)=0$ and $\mathbb{E} \varepsilon_{1}^{2}<\infty$ almost sure convergence of (9) follows from Cuzick (1995, Theorem 1.1) or Choi and Sung (1987, Theorem 5).

It remains to show that

$$
\begin{aligned}
& \frac{1}{T} \sum_{j=0}^{\infty} \tilde{\psi}_{j}(1) \varepsilon_{T-j} \stackrel{a . s .}{\rightarrow} 0, \\
& \frac{1}{T} \sum_{j=0}^{\infty} \tilde{\psi}_{j}(0) \varepsilon_{-j} \stackrel{\text { a.s. }}{\rightarrow} 0
\end{aligned}
$$

and

$$
\frac{1}{T} \sum_{t=1}^{T} \sum_{j=0}^{\infty}\left\{\tilde{\psi}_{j}\left(\frac{t-1}{T}\right)-\tilde{\psi}_{j}\left(\frac{t}{T}\right)\right\} \varepsilon_{t-1-j} \stackrel{a . s .}{\rightarrow} 0 .
$$

It holds that

$$
\begin{aligned}
\mathbb{E}\left(\frac{1}{T} \sum_{j=0}^{\infty} \tilde{\psi}_{j}(1) \varepsilon_{T-j}\right)^{2} & =\frac{1}{T^{2}} \sum_{j=0}^{\infty} \tilde{\psi}_{j}(1)^{2} \\
& =\frac{1}{T^{2}} \sum_{j=0}^{\infty}\left(\sum_{i=j+1}^{\infty} \psi_{i}(1)\right)^{2} \\
& =\mathcal{O}\left(\frac{1}{T^{2}}\right)
\end{aligned}
$$


since

$$
\sum_{j=0}^{\infty} \sum_{i=j+1}^{\infty} \psi_{i}(1) \leq \sum_{j=0}^{\infty} \sum_{i=j+1}^{\infty}\left\|\psi_{i}\right\|_{\infty}=\sum_{j=0}^{\infty} j\left\|\psi_{j}\right\|_{\infty}<\infty
$$

Hence, the term in (10) converges sufficiently fast to zero in probability and almost sure convergence follows from the Borel-Cantelli lemma. The proof of (11) is identical. At last we have to show (12). It holds that

$$
\begin{aligned}
& \mathbb{E}\left(\frac{1}{T} \sum_{t=1}^{T} \sum_{j=0}^{\infty}\left\{\tilde{\psi}_{j}\left(\frac{t-1}{T}\right)-\tilde{\psi}_{j}\left(\frac{t}{T}\right)\right\} \varepsilon_{t-1-j}\right)^{2} \\
& \quad \leq \frac{1}{T^{2}} \sum_{j_{1}, j_{2}=0}^{\infty} \sum_{t_{1}, t_{2}=1}^{T}\left|\tilde{\psi}_{j_{1}}\left(\frac{t_{1}-1}{T}\right)-\tilde{\psi}_{j_{1}}\left(\frac{t_{1}}{T}\right)\right|\left|\tilde{\psi}_{j_{2}}\left(\frac{t_{2}-1}{T}\right)-\tilde{\psi}_{j_{2}}\left(\frac{t_{2}}{T}\right)\right| \\
& \quad \leq \frac{1}{T^{2}} \sum_{j=0}^{\infty} V\left(\tilde{\psi}_{j}\right) \sum_{k=0}^{\infty} V\left(\tilde{\psi}_{k}\right)=\mathcal{O}\left(\frac{1}{T^{2}}\right) .
\end{aligned}
$$

Hence, the second moment of the term in (12) converges sufficiently fast to zero implying almost sure convergence.

Our last result is a LIL. In order to prove the theorem we impose some additional moment condition on the sequence $\left\{\varepsilon_{t}\right\}_{t \in \mathbb{Z}}$. In particular, we assume that at least the fourth moment of $\varepsilon_{t}$ is finite.

Theorem 2.5 (LIL) Let $\left\{\eta_{t, T}\right\}_{t=1, \ldots, T}$ be a locally stationary process with Assumptions 1.1, 1.3 and 2.3 in place and let $d_{T}=T \log \log T$. Assume further that the innovation sequence $\left\{\varepsilon_{t}\right\}_{t \in \mathbb{Z}}$ satisfies $\mathbb{E} \varepsilon_{t}^{4}=\mu_{4}<\infty$. Then, as $T \rightarrow \infty$, it holds that

$$
\limsup _{T \rightarrow \infty} \frac{1}{\sqrt{d_{T}}} \sum_{t=1}^{T}\left\{\eta_{t, T}-\mu\left(\frac{t}{T}\right)\right\} \stackrel{a . s .}{=} \sqrt{2}\|\Psi\|_{L^{2}} .
$$

Proof By Lemma A.3 it suffices to show the claim for the auxiliary process $\left\{\tilde{\eta}_{t, T}\right\}_{t=1, \ldots, T}$. Following the lines of the proof of Theorem 2.4 we first prove that

$$
\limsup _{T \rightarrow \infty} \frac{1}{\sqrt{d_{T}}} \sum_{t=1}^{T} \Psi\left(\frac{t}{T}\right) \varepsilon_{t} \stackrel{\text { a.s. }}{=} \sqrt{2}\|\Psi\|_{L^{2}} .
$$

Since $\left\{\varepsilon_{t}\right\}_{t \in \mathbb{Z}}$ is a sequence of independent random variables with finite variance the claim follows immediately from Tomkins (1975, Theorem 1), Wichura (1973, page 279) and Lai and Wei (1982, Corollary 2). Therefore, it remains to prove that 


$$
\begin{aligned}
& \frac{1}{\sqrt{d_{T}}} \sum_{j=0}^{\infty} \tilde{\psi}_{j}(1) \varepsilon_{T-j} \stackrel{\text { a.s. }}{\rightarrow} 0, \\
& \frac{1}{\sqrt{d_{T}}} \sum_{j=0}^{\infty} \tilde{\psi}_{j}(0) \varepsilon_{-j} \stackrel{\text { a.s. }}{\rightarrow} 0
\end{aligned}
$$

and

$$
\frac{1}{\sqrt{d_{T}}} \sum_{t=1}^{T} \sum_{j=0}^{\infty}\left(\tilde{\psi}_{j}\left(\frac{t-1}{T}\right)-\tilde{\psi}_{j}\left(\frac{t}{T}\right)\right) \varepsilon_{t-1-j} \stackrel{a . s .}{\rightarrow} 0 .
$$

In contrast to the proof of Theorem 2.4 it is not sufficient to investigate the second moments of these terms, as $d_{T}^{-1}$ decays too slowly. However, we adapt the proof using fourth moments. For the term in (13) it holds that

$$
\begin{aligned}
\mathbb{E}\left(\sum_{j=0}^{\infty} \tilde{\psi}_{j}(1) \varepsilon_{T-j}\right)^{4} & =\sum_{j_{1}, \ldots, j_{4}=0}^{\infty}\left\{\prod_{m=1}^{4} \tilde{\psi}_{j_{m}}(1)\right\} \mathbb{E}\left(\prod_{m=1}^{4} \varepsilon_{T-j_{m}}\right) \\
& \leq \mu_{4}\left(\sum_{j=0}^{\infty} \tilde{\psi}_{j}(1)\right)^{4}
\end{aligned}
$$

implying

$$
\sum_{T=1}^{\infty} \mathbb{E}\left(\frac{1}{\sqrt{d_{T}}} \sum_{j=0}^{\infty} \tilde{\psi}_{j}(1) \varepsilon_{T-j}\right)^{4} \leq \sum_{T=1}^{\infty} \frac{C}{T^{2}(\log \log T)^{2}}<\infty
$$

and, by the Borel-Cantelli lemma, almost sure convergence. The claim in (14) is proven in exactly the same way. To show (15) consider

$$
\begin{aligned}
& \mathbb{E}\left(\frac{1}{\sqrt{d_{T}}} \sum_{t=1}^{T} \sum_{j=0}^{\infty}\left(\tilde{\psi}_{j}\left(\frac{t-1}{T}\right)-\tilde{\psi}_{j}\left(\frac{t}{T}\right)\right) \varepsilon_{t-1-j}\right)^{4} \\
& \quad=\frac{1}{d_{T}^{2}} \sum_{t_{1}, \ldots, t_{4}=1}^{T} \sum_{j_{1}, \ldots, j_{4}=0}^{\infty}\left\{\prod_{m=1}^{4}\left(\tilde{\psi}_{j}\left(\frac{t-1}{T}\right)-\tilde{\psi}_{j}\left(\frac{t}{T}\right)\right)\right\} \mathbb{E}\left(\prod_{m=1}^{4} \varepsilon_{t_{m}-1-j_{m}}\right) \\
& \quad \leq \frac{1}{d_{T}^{2}} \sum_{j_{1}, \ldots, j_{4}=0}^{\infty}\left\{\prod_{m=1}^{4} \sum_{t_{m}=1}^{T}\left|\tilde{\psi}_{j}\left(\frac{t_{m}-1}{T}\right)-\tilde{\psi}_{j}\left(\frac{t_{m}}{T}\right)\right|\right\} \mu_{4} \\
& \quad \leq \frac{1}{d_{T}^{2}} \sum_{j_{1}, \ldots, j_{4}=0}^{\infty}\left\{\prod_{m=1}^{4} V\left(\tilde{\psi}_{j_{m}}\right)\right\}=\frac{1}{d_{T}^{2}}\left(\sum_{j=0}^{\infty} V\left(\tilde{\psi}_{j}\right)\right)^{4} \mu_{4} .
\end{aligned}
$$

The claim follows by the same arguments as above. 
Obviously, if the coefficients $\psi_{j, t, T}$ are not time-dependent, the statement of Theorem 2.5 coincides with the LIL for linear processes which was proven by Phillips and Solo (1992).

Acknowledgements This work has been supported in part by the Collaborative Research Center Statistical modeling of nonlinear dynamic processes (SFB 823, Teilprojekt A3, A4) of the German Research Foundation (DFG).

Funding Open Access funding enabled and organized by Projekt DEAL.

Open Access This article is licensed under a Creative Commons Attribution 4.0 International License, which permits use, sharing, adaptation, distribution and reproduction in any medium or format, as long as you give appropriate credit to the original author(s) and the source, provide a link to the Creative Commons licence, and indicate if changes were made. The images or other third party material in this article are included in the article's Creative Commons licence, unless indicated otherwise in a credit line to the material. If material is not included in the article's Creative Commons licence and your intended use is not permitted by statutory regulation or exceeds the permitted use, you will need to obtain permission directly from the copyright holder. To view a copy of this licence, visit http://creativecommons.org/licenses/by/4.0/.

\section{Appendix: Auxiliary lemmata}

Lemma A.1 Let $\left\{\eta_{t, T}\right\}_{t=1, \ldots, T}$ be a locally stationary process defined via its moving average representation (1) with Assumptions 1.1 and 1.3 in place and let $\left\{\tilde{\eta}_{t, T}\right\}_{t=1, \ldots, T}$ be the corresponding auxiliary process, defined by (3). Then it holds that

$$
\sum_{t=1}^{T}\left|\eta_{t, T}-\tilde{\eta}_{t, T}\right|=\mathcal{O}_{P}(1)
$$

Proof It holds that

$$
\sum_{t=1}^{T}\left|\eta_{t, T}-\tilde{\eta}_{t, T}\right| \leq \sum_{j=0}^{\infty} \sum_{t=1}^{T}\left|\psi_{j, t, T}-\psi_{j}\left(\frac{t}{T}\right)\right|\left|\varepsilon_{t-j}\right|
$$

Hence,

$$
\sum_{t=1}^{T} \mathbb{E}\left|\eta_{t, T}-\tilde{\eta}_{t, T}\right| \leq \sum_{j=0}^{\infty} \sum_{t=1}^{T}\left|\psi_{j, t, T}-\psi_{j}\left(\frac{t}{T}\right)\right| \mathbb{E}\left|\varepsilon_{1}\right| \leq \sum_{j=0}^{\infty} \frac{K \mathbb{E}\left|\varepsilon_{1}\right|}{l(j)}<\infty
$$

implying the claim.

Lemma A.2 Let $\left\{\eta_{t, T}\right\}_{t=1, \ldots, T}$ be a locally stationary process defined via its moving average representation (1) with Assumptions 1.1 and 2.3 in place. Further, let $\left\{\tilde{\eta}_{t, T}\right\}_{t=1, \ldots, T}$ be the corresponding auxiliary process, defined by (3). Then, it holds that 


$$
\sup _{1 \leq t \leq T}\left|\eta_{t, T}-\tilde{\eta}_{t, T}\right| \stackrel{\text { a.s. }}{\rightarrow} 0
$$

as $T \rightarrow \infty$.

Proof It holds that

$$
\left|\eta_{t, T}-\tilde{\eta}_{t, T}\right| \leq \sum_{j=0}^{\infty}\left|\psi_{j, t, T}-\psi_{j}\left(\frac{t}{T}\right)\right|\left|\varepsilon_{t-j}\right| \leq \frac{1}{T} \sum_{j=0}^{\infty} \frac{K}{l(j)}\left|\varepsilon_{t-j}\right| .
$$

Using Hölder's inequality we obtain

$$
\begin{aligned}
\sum_{j=0}^{\infty} \frac{K}{l(j)}\left|\varepsilon_{t-j}\right| & =\sum_{j=0}^{\infty}\left(\frac{K}{l(j)}\right)^{\frac{1+\kappa}{2+\kappa}}\left(\frac{K}{l(j)}\right)^{\frac{1}{2+\kappa}}\left|\varepsilon_{t-j}\right| \\
& \leq\left(\sum_{j=0}^{\infty} \frac{K}{l(j)}\right)^{\frac{1+\kappa}{2+\kappa}}\left(\sum_{j=0}^{\infty} \frac{K}{l(j)}\left|\varepsilon_{t-j}\right|^{2+\kappa}\right)^{\frac{1}{2+\kappa}}
\end{aligned}
$$

This implies that

$$
\begin{aligned}
\mathbb{E}\left|\eta_{t, T}-\tilde{\eta}_{t, T}\right|^{2+\kappa} & \leq \mathbb{E}\left(\frac{1}{T} \sum_{j=0}^{\infty} \frac{K}{l(j)}\left|\varepsilon_{t-j}\right|\right)^{2+\kappa} \\
& \leq \frac{1}{T^{2+\kappa}}\left(\sum_{j=0}^{\infty} \frac{K}{l(j)}\right)^{1+\kappa} \sum_{j=0}^{\infty} \frac{K}{l(j)} \mathbb{E}\left|\varepsilon_{t-j}\right|^{2+\kappa} \\
& =\frac{\tilde{K}}{T^{2+\kappa}}
\end{aligned}
$$

since, by Assumption 1.1, $\left\{\varepsilon_{t}\right\}_{t \in \mathbb{Z}}$ is a sequence of independent random variables and there exists some $\kappa>0$ such that $\mathbb{E}\left|\varepsilon_{t}\right|^{2+\kappa}<\infty$. Hence, for all $\alpha>0$ we obtain, applying the Bonferroni and the Markov inequalities,

$$
\begin{aligned}
\sum_{T=1}^{\infty} P\left(\sup _{1 \leq t \leq T}\left|\eta_{t, T}-\tilde{\eta}_{t, T}\right|>\alpha\right) & \leq \sum_{T=1}^{\infty} \sum_{t=1}^{T} P\left(\left|\eta_{t, T}-\tilde{\eta}_{t, T}\right|>\alpha\right) \\
& \leq \sum_{T=1}^{\infty} \sum_{t=1}^{T} \frac{\mathbb{E}\left|\eta_{t, T}-\tilde{\eta}_{t, T}\right|^{2+\kappa}}{\alpha^{2+\kappa}} \\
& \leq \sum_{T=1}^{\infty} \sum_{t=1}^{T} \frac{\tilde{K}}{(\alpha T)^{2+\kappa}} \\
& =\sum_{T=1}^{\infty} \frac{\tilde{K}}{\alpha^{2+\kappa} T^{1+\kappa}}<\infty
\end{aligned}
$$

which implies the claim. 
Lemma A.3 Let $\left\{\eta_{t, T}\right\}_{t=1, \ldots, T}$ be a locally stationary process defined via its moving average representation (1) with corresponding auxiliary process $\left\{\tilde{\eta}_{t, T}\right\}_{t=1, \ldots, T}$, defined by (3). Under the Assumptions of Theorem 2.5 it holds that

$$
\frac{1}{\sqrt{d_{T}}} \sum_{t=1}^{T}\left(\eta_{t, T}-\tilde{\eta}_{t, T}\right) \stackrel{\text { a.s. }}{\rightarrow} 0
$$

as $T \rightarrow \infty$, where $d_{T}=T \log \log T$.

Proof It holds that

$\frac{1}{\sqrt{d_{T}}} \sum_{t=1}^{T}\left|\eta_{t, T}-\tilde{\eta}_{t, T}\right| \leq \frac{1}{\sqrt{d_{T}}} \sum_{t=1}^{T} \sup _{1 \leq t \leq T}\left|\eta_{t, T}-\tilde{\eta}_{t, T}\right| \leq \frac{T}{\sqrt{d_{T}}} \sup _{1 \leq t \leq T}\left|\eta_{t, T}-\tilde{\eta}_{t, T}\right|$.

From the proof of Lemma A.2 we deduce that for $\alpha>0$

$$
\begin{aligned}
\sum_{T=1}^{\infty} P\left(\frac{T}{\sqrt{d_{T}}} \sup _{1 \leq t \leq T}\left|\eta_{t, T}-\tilde{\eta}_{t, T}\right|>\alpha\right) & \leq \sum_{T=1}^{\infty} \sum_{t=1}^{T} \frac{\tilde{K} T^{2+\kappa}}{\alpha^{2+\kappa} T^{2+\kappa} d_{T}^{1+\kappa / 2}} \\
& =\frac{\tilde{K}}{\alpha^{2+\kappa}} \sum_{T=1}^{\infty} \frac{T}{d_{T}^{1+\kappa / 2}} .
\end{aligned}
$$

Since $\mathbb{E} \varepsilon_{t}^{4}=\mu_{4}$ is finite by assumption we can set $\kappa=2$ and obtain

$$
\sum_{T=1}^{\infty} \frac{T}{d_{T}^{1+2 / 2}}=\sum_{T=1}^{\infty} \frac{T}{(T \log \log T)^{2}}=\sum_{T=1}^{\infty} \frac{1}{T(\log \log T)^{2}}<\infty
$$

which yields almost sure convergence.

\section{References}

Choi BD, Sung SH (1987) Almost sure convergence theorems for weighted sums of random variables. Stoch Anal Appl 5(4):365-377

Cuzick J (1995) A strong law of for weighted sums of i.i.d. random variables. J Theor Probab 8(3):625-641 Dahlhaus R (2012) Locally stationary processes. In: Rao TS, Rao SS, Rao C (eds) Handbook of statistics, time series analysis: methods and applications, vol 30, Elsevier, chap 13, pp 351-413

Dahlhaus R, Polonik W (2006) Nonparametric quasi-maximum likelihood estimation for gaussian locally stationary processes. Ann Stat 34(6):2790-2824

Dahlhaus R, Polonik W (2009) Empirical spectral processes for locally stationary time series. Bernoulli 15(1):1-39

Künsch HR (1995) A note on causal solutions for locally stationary ar-processes, preprint ETH Zürich

Lai TL, Wei CZ (1982) A law of the iterated logarithm for double arrays of independent random variables with applications to regression and time series models. Ann Probab 10(2):320-335

Phillips PC, Solo V (1992) Asymptotics for linear processes. Ann Stat 20(2):971-1001

Tomkins RJ (1975) Iterated logarithm results for weighted averages of martingale difference sequences. Ann Probab 3(2):307-314 
Wichura MJ (1973) Some Strassen-type laws of the iterated logarithm for multiparameter stochastic processes with independent increments. Ann Probab 1(2):272-296

Publisher's Note Springer Nature remains neutral with regard to jurisdictional claims in published maps and institutional affiliations. 